\title{
Effects of RGE on fermion observables in S010 models
}

\author{
Davide Meloni* \\ Dipartimento di Matematica e Fisica, Roma Tre University, via della Vasca Navale 84, 00146 \\ Roma \\ E-mail: davide.meloni@uniroma3.it
}

In the context of non-supersymmetric $\mathrm{SO}(10)$ models, we analyze the renormalization group equations for fermions from the GUT energy scale down to the electroweak energy scale, explicitly taking into account the effects of an intermediate energy scale induced by a Pati-Salam gauge group. To determine the renormalization group running, we use a numerical minimization procedure based on a nested sampling algorithm that randomly generates the values of 19 model parameters at the GUT scale, evolves them, and finally constructs the values of the physical observables and compares them to the existing experimental data at the electroweak scale. We show that the evolved fermion masses and mixings present sizable deviations from the values obtained without including the effects of the intermediate scale.

The European Physical Society Conference on High Energy Physics

5-12 July, 2017

Venice

${ }^{*}$ Speaker. 


\section{Introduction}

The lack of signals from physics beyond the Standard Model (SM) at the Large Hadron Collider (LHC) revives the question of which model constitutes the most appropriate extension of the SM and, if there is one, what is the energy scale where new features of particle interactions ought to be observed. In the present talk, we will study a non-supersymmetric extension of the SM model based on the gauge group $\mathrm{SO}(10)$ with the intent to check the ability to reproduce the values of fermion masses and mixings at the electroweak (EW) scale $M_{Z}$, taking into account the effects of an intermediate energy scale in the Yukawa RGE's.

We quantify the impact of using such new contributions in the renormalization group equations (RGEs) for fermion masses and mixings, considering an illustrative and simplified $\mathrm{SO}(10)$ model with a breaking chain given by $[1,2]$ :

$$
\mathrm{SO}(10) \stackrel{M_{\mathrm{GUT}}-210_{\mathrm{H}}}{\longrightarrow} 4_{\mathrm{C}} 2_{\mathrm{L}} 2_{\mathrm{R}} \stackrel{M_{\mathrm{I}}-126_{\mathrm{H}}}{\longrightarrow} 3_{\mathrm{C}} 2_{\mathrm{L}} 1_{Y} \stackrel{M_{\mathrm{Z}}-10_{\mathrm{H}}}{\longrightarrow} 3_{\mathrm{C}} 1_{\mathrm{Y}}
$$

where the symbols are self-explanatory. The breaking of $\mathrm{SO}(10)$ down to the PS group at the GUT scale $M_{\mathrm{GUT}}$ is achieved by means of a $210_{\mathrm{H}}$ representation of Higgs. In the next step, the breaking of the PS group down to the SM gauge group is performed by means of a $\overline{126}_{\mathrm{H}}$. At $M_{\mathrm{Z}}$, the final step of the breaking of the $\mathrm{SM}$ gauge group to $\mathrm{SU}(3)_{\mathrm{C}} \times \mathrm{U}(1)_{\mathrm{Y}}$ is obtained with a $10_{\mathrm{H}}$.

The output of our analysis will be the values of the elements of the Yukawa matrices at $M_{\mathrm{GUT}}$, which give a reasonable fit to the fermion observables at $M_{Z}$. These values can directly be compared to the corresponding ones obtained from an evolution without the intermediate scale starting at $M_{\mathrm{GUT}}$, thus allowing a quantification of the new effects introduced by the PS gauge group [3,4].

\section{Evolution of gauge coupling constants and Yukawas}

In the PS group, the Higgs and matter fields decompose as:

$$
\begin{aligned}
10_{\mathrm{H}} & =(1,2,2) \oplus(6,1,1), \quad 16=(4,2,1) \oplus(\overline{4}, 1,2) \equiv F_{\mathrm{L}}+F_{\mathrm{R}} \\
\overline{126}_{\mathrm{H}} & =(6,1,1) \oplus(10,1,3) \oplus(\overline{10}, 3,1) \oplus(15,2,2),
\end{aligned}
$$

where $F_{\mathrm{L}}$ and $F_{\mathrm{R}}$ are the left- and right-handed parts of the 16 , respectively. At $M_{\mathrm{GUT}}$, the gauge couplings are unified and the matching conditions are simply $\alpha_{4 \mathrm{C}}\left(M_{\mathrm{GUT}}\right)=\alpha_{2 \mathrm{R}}\left(M_{\mathrm{GUT}}\right)=\alpha_{2 \mathrm{~L}}^{\prime}\left(M_{\mathrm{GUT}}\right)$, where $\alpha_{4 \mathrm{C}}, \alpha_{2 \mathrm{R}}$, and $\alpha_{2 \mathrm{~L}}^{\prime}$ are the coupling constants of $\mathrm{SU}(4), \mathrm{SU}(2)_{\mathrm{R}}$, and $\mathrm{SU}(2)_{\mathrm{L}}$ above $M_{\mathrm{I}}$, respectively. For $a_{4 \mathrm{C}}, a_{2 \mathrm{~L}}^{\prime}$, and $a_{2 \mathrm{R}}$ between $M_{\mathrm{GUT}}$ and $M_{\mathrm{I}}$ the matching conditions are

$$
\alpha_{3 \mathrm{C}}\left(M_{\mathrm{I}}\right)=\alpha_{4 \mathrm{C}}\left(M_{\mathrm{I}}\right), \quad \alpha_{2 L}\left(M_{\mathrm{I}}\right)=\alpha_{2 \mathrm{~L}}^{\prime}\left(M_{\mathrm{I}}\right), \quad \alpha_{1 \mathrm{Y}}^{-1}\left(M_{\mathrm{I}}\right)=\frac{3}{5} \alpha_{2 \mathrm{R}}^{-1}\left(M_{\mathrm{I}}\right)+\frac{2}{5} \alpha_{4 \mathrm{C}}^{-1}\left(M_{\mathrm{I}}\right),
$$

where $\alpha_{3 \mathrm{C}}, \alpha_{2 \mathrm{~L}}$, and $\alpha_{1 \mathrm{Y}}$ are the SM gauge coupling constants. Eventually, in the running from $M_{\mathrm{I}}$ down to $M_{\mathrm{Z}}$, four Higgs representations are involved, stemming from the bidoublet of the $10_{\mathrm{H}}$ and $126_{\mathrm{H}}$. The values of the mass scales obtained solving the one-loop RGE's for the gauge couplings are $M_{\mathrm{I}}=(1.5 \pm 0.2) \cdot 10^{12} \mathrm{GeV}$ and $M_{\mathrm{GUT}}=(1.7 \pm 0.6) \cdot 10^{15} \mathrm{GeV}$. 
For the Yukawa sector, at $M_{\mathrm{GUT}}$ we have the following Lagrangian:

$$
L_{Y}=16\left(h 10_{\mathrm{H}}+f \overline{126}_{\mathrm{H}}\right) 16,
$$

where $h$ and $f$ are unknown symmetric couplings to be determined through a fitting procedure. In the region between $M_{\mathrm{GUT}}$ and $M_{\mathrm{I}}$, the Yukawa part of the Lagrangian is given by [5]

$$
-\mathscr{L}_{\mathrm{Y}}=\sum_{i, j}\left(Y_{F i j}^{(10)} F_{\mathrm{L}}^{i T} \Phi F_{\mathrm{R}}^{j}+Y_{F i j}^{(126)} F_{\mathrm{L}}^{i T} \Sigma F_{\mathrm{R}}^{j}+Y_{R i j}^{(126)} F_{\mathrm{R}}^{i T} \overline{\Delta_{R}} F_{\mathrm{R}}^{j}+\text { h.c. }\right),
$$

where $Y_{F}^{(10)}, Y_{F}^{(126)}$, and $Y_{R}^{(126)}$ are Yukawa couplings and we have used he short-hand notation

$$
\Phi \equiv(1,2,2), \quad \Sigma \equiv(15,2,2), \quad \overline{\Delta_{R}} \equiv(10,1,3) .
$$

Furthermore, at $M_{\mathrm{GUT}}$, the couplings $Y_{F}^{(10)}, Y_{F}^{(126)}$, and $Y_{R}^{(126)}$ have to be matched to $h$ and $f$ :

$$
\frac{1}{\sqrt{2}} Y_{F}^{(10)}\left(M_{\mathrm{GUT}}\right) \equiv h, \quad \frac{1}{4 \sqrt{2}} Y_{F}^{(126)}\left(M_{\mathrm{GUT}}\right)=\frac{1}{4} Y_{R}^{(126)}\left(M_{\mathrm{GUT}}\right) \equiv f,
$$

where the numerical factors are Clebsch-Gordan coefficients needed for a correct embedding of PS into SO(10) [6]. Below this scale we are left with the SM Lagrangian with four Higgses. The resulting mass matrices, including the ones for neutrinos, can be found in [3].

\section{Numerical parameter-fitting procedure and results}

We adopt the procedure of considering the entries of the couplings $h$ and $f$ as well as the vacuum expectation values of the fields in 2.5 as our free parameters and evolving them down to the EW scale $M_{\mathrm{Z}}$, where the values of masses and mixings of quarks, charged leptons, and neutrinos are known. There are in total 19 free parameters at $M_{\mathrm{GUT}}$ which need to be determined (including one Higgs self-coupling at $M_{\mathrm{I}}$, not discussed here). To evolve the Yukawa's we first randomly generate the values of the parameters at $M_{\mathrm{GUT}}$, according to some prior distribution; then, we evolve them down to $M_{\mathrm{Z}}$ after solving the RGEs; next, at $M_{\mathrm{Z}}$, we construct the observables and compare them to the experimental data. The procedure is repeated with new randomly sampled parameter values from a reduced parameter space and the result is given when convergence on the point with largest likelihood occurs, i.e. on the best-fit point.

For the sampling procedure, we used the software MultiNest, which is based on nested sampling normally used for calculation of the Bayesian evidence [7]. The experimental values of the quark and charged lepton masses are taken from Ref. [8], the quark mixing parameters from Ref. [9], and the neutrino mass-squared differences and the leptonic mixing angles from Ref. [10].

The effect of $M_{\mathrm{I}}$ on the RG running is appreciated by comparing such values with the ones obtained from RG running without $M_{\mathrm{I}}$. For the up and down-type quarks, this is reported in Fig. 1. In the case of the model with $M_{\mathrm{I}}$, we observe that the slope of the RG running of the quark masses changes direction at $M_{\mathrm{I}}$ : from $M_{\mathrm{GUT}}$ down to $M_{\mathrm{I}}$, it decreases monotonically, whereas from $M_{\mathrm{I}}$ down to $M_{\mathrm{Z}}$, it increases monotonically. The reason for this change of direction in the evolution can be deduced from the change of sign in front of the gauge coupling terms, which dominate the $\beta$-functions in the RGEs that are given in [3]. As expected, in the case of the model without $M_{\mathrm{I}}$, 

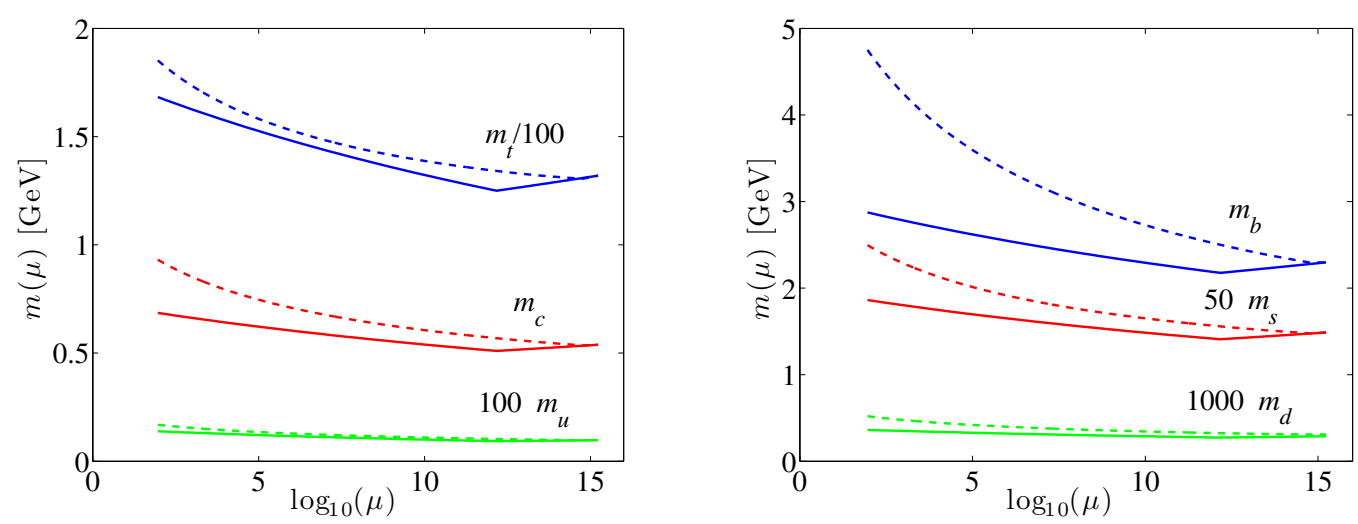

Figure 1: The RG running of the up-type (left plot) and down-type (right plot) quark masses, with (solid curves) and without (dashed curves) the intermediate energy scale $M_{\mathrm{I}}$ as functions of the energy scale $\mu$.

i.e. the $\mathrm{SM}$ case, the RG running from $M_{\mathrm{GUT}}$ down to $M_{\mathrm{Z}}$ increases monotonically. Thus, at the $M_{\mathrm{Z}}$, the quark masses in the two cases will differ, and they will be larger in the SM case than in the model with $M_{\mathrm{I}}$. The smallest difference is for the top quark mass, which is $10 \%$ larger at $M_{\mathrm{Z}}$, whereas the largest difference is for the bottom quark mass, which is $65 \%$ larger.

\section{Summary and conclusions}

In conclusion, we have explored the effects of an intermediate energy scale on the evolution of the fermion masses and mixings in an $\mathrm{SO}(10)$ model with a Pati-Salam intermediate gauge group. The effects have been compared to the evolution from the GUT scale down to the EW scale in a SM-like model with two Higgs doublets. We have found that the solutions to the RGEs, i.e., the values of the fermion observables, at the EW scale in the SM, disagree compared to the $\mathrm{SO}(10)$ model well beyond experimental uncertainties, which are at the level of $30 \%$ for the quark masses.

\section{References}

[1] N. Deshpande, E. Keith, and P. B. Pal, Phys. Rev. D46 (1993) 2261-2264.

[2] G. Altarelli and D. Meloni, JHEP 1308 (2013) 021.

[3] D. Meloni, T. Ohlsson and S. Riad, JHEP 1412 (2014) 052.

[4] D. Meloni, T. Ohlsson and S. Riad, JHEP 1703 (2017) 045.

[5] T. Fukuyama and T. Kikuchi, Mod. Phys. Lett. A18 (2003) 719-731.

[6] C. S. Aulakh and A. Girdhar, Int. J. Mod. Phys. A20 (2005) 865-894.

[7] F. Feroz and M. Hobson, Mon. Not. Roy. Astron. Soc. 384 (2008) 449-463.

[8] Z.-z. Xing, H. Zhang, and S. Zhou, Phys. Rev. D77 (2008) 113016.

[9] A. S. Joshipura and K. M. Patel, Phys. Rev. D83 (2011) 095002.

[10] M. Gonzalez-Garcia, M. Maltoni, J. Salvado, and T. Schwetz, JHEP 1212 (2012) 123. 